\title{
circSMARCA5-SRSF1 interaction is functionally involved in GBM pathogenesis*
}

\author{
Angela Caponnetto ${ }^{1 \dagger}$ \\ ${ }^{1}$ Department of Biomedical and Biotechnological Sciences, Section of Biology and \\ Genetics 'Giovanni Sichel', University of Catania, I-95123 Catania, Italy
}

\section{Summary}

Glioblastoma multiforme (GBM) is the most aggressive human brain tumor with a median survival of 15 months. The standard treatments of GBM and the total medical resection are unable to contrast this mortal cancer. For these reason new diagnostic approaches and therapies are needed. The identification of molecular features of this cancer may allow to create a personalized therapy. Circular RNAs (circRNAs) are a new class of non-coding RNAs (ncRNAs) highly enriched in brain, stable within the cells, detectable in body fluids and having a potential role and biological importance still object of debate. This thesis investigated the putative involvement of circRNAs in GBM pathogenesis. It has been demonstrated that circSMARCA5 is downregulated in GBM biopsies, its expression is associated to the glioma grade malignancy and it negatively regulates migration of U87MG cells. Moreover, it has been proved the physical interaction between circSMARCA5 and one of its predicted interactor Serine/argininerich splicing factor 1 (SRSF1). Interesting splicing targets of SRSF1 are the serine and arginine rich splicing factor 3 (SRSF3) and the vascular endothelial growth factor A (VEGFA). It has been proposed that circSMARCA5 may regulate the alternative splicing of SRSF3 in favor of the formation of a stable oncoprotein in GBM. It also regulates the alternative splicing of VEGFA mRNA through the binding to SRSF1. In addition, blood vascular microvessel density evaluated in GBM negatively correlates with the expression of circSMARCA5, while positively correlates with that of SRSF1 and pro-toanti-angiogenic VEGFA isoform ratio. GBM patients with low circSMARCA5 expression have lower overall and progression free survival rates. Based on these findings, CircSMARCA5 could be considered a promising druggable tumor suppressor in GBM. Moreover, its interaction with SRSF1 makes circSMARCA5 an upstream regulator of pro- to

*Abstract of one of the Best PhD Theses Award, 2021.

†E-mail: angela.caponnetto@unict.it 
anti-angiogenic VEGFA isoforms ratio within GBM cells and a highly promising GBM prospective anti-angiogenic molecule.

Keywords: glioblastoma multiforme, circular RNAs, alternative splicing, angiogenesis.

\section{Riassunto}

\section{L'interazione tra circSMARCA5 ed SRSF1 è funzionalmente coinvolta nella patogenesi del GBM*}

Il Glioblastoma multiforme (GBM) è uno dei tumori cerebrali umani più aggressivi con una sopravvivenza media di circa 15 mesi. I trattamenti standard del GBM e la resezione chirurgica non sono in grado di contrastare questo tumore mortale. Per queste ragioni, sono necessari nuovi approcci diagnostici e terapeutici. L'identificazione di caratteristiche molecolari di questo tumore permetterebbe la creazione di terapie personalizzate. Gli RNA circolari sono una classe di RNA non codificanti altamente abbondanti nel cervello, stabili all'interno delle cellule, identificabili nei fluidi corporei ed aventi un potenziale ruolo e importanza biologica ad oggi oggetto di dibattito. Questo lavoro di tesi è focalizzato sullo studio del potenziale coinvolgimento degli RNA circolari nella patogenesi del GBM. È stato dimostrato che l'RNA circolare SMARCA5 (circSMARCA5) è sotto-espresso nelle biopsie di GBM, la sua espressione è associata al grado di malignità tumorale e regola negativamente la migrazione di cellule U87MG. Inoltre, è stata provata la fisica interazione tra circCMARCA5 ed uno dei suoi bersagli predetti, il fattore di splicing Serine/arginine-rich splicing factor 1 (SRSF1). Interessanti bersagli di splicing di SRSF1 sono serine and arginine rich splicing factor 3 (SRSF3) e il vascular endothelial growth factor $A$ (VEGFA). È stato proposto che circSMARCA5 può regolare lo splicing alternativo di SRSF3 favorendo la produzione di una oncoproteina stabile nel GBM. Esso inoltre regola lo splicing alternativo di VEGFA attraverso il legame con SRS1. In aggiunta, la densità microvascolare, valutata nei campioni GBM, è negativamente correlata con l'espressione di circSMARCA5, mentre è positivamente correlata con l'espressione di SRSF1 e con il rapporto delle isoforme pro-angiogenica/anti-angiogenica di VEGFA. Infine, i pazienti affetti da GBM con una minore espressione di circSMARCA5 hanno una sopravvivenza media e una sopravvivenza priva di progressione della malattia inferiori. Sulla base di questi risultati, circSMARCA5 potrebbe essere considerato un promettente soppressore tumorale. Inoltre, la sua interazione con SRSF1 rende circSMARCA5 un regolatore a monte del rapporto isoforma pro-angiogenica/anti-angiogenica di VEGFA nelle cellule di GBM e una molecola ad azione anti-angiogenica altamente promettente.

Parole chiave: glioblastoma multiforme, RNA circolari, splicing alternativo, angiogenesi.

*Riassunto di una delle Migliori Tesi di Dottorato premiate nel 2021. 